\title{
Prevalência de erros refrativos em escolares da primeira série do ensino fundamental da região Nordeste do Rio Grande do Sul
}

\author{
Prevalence of refractive errors in first grade school children \\ of elementary schools of Northeast region of the \\ Rio Grande do Sul State, Brazil
}

Paulo Estacia', Leandro Mazzoleni Stramari², Silvia Bassani Schuch², Danielle Negrello², Luciane Donato²

\section{ResUMO}

Objetivos: Descrever e analisar a prevalência de erros refrativos em estudantes de primeira série das escolas estaduais de Passo Fundo, cidade pólo da região Nordeste do Rio Grande do Sul. Métodos: Estudo transversal de 1608 alunos da primeira série do ensino fundamental de 26 escolas estaduais de Passo Fundo, atendidas em 2004. Os dados foram coletados no CEAE (Centro Especializado de Assistência ao Educando), que fez a triagem nas escolas, e no serviço especializado, para onde foram encaminhados aqueles com testes alterados.Resultados: Da amostra total,172 (10,88\%) apresentaram baixa de acuidade visual ou algum sintoma de problema visual, recebendo encaminhamento para atendimento oftalmológico. Apenas 88 (51,16\%) dos encaminhados compareceram à consulta. A média de idade foi de 7,10 anos $(\mathrm{DP}=1,38)$. Entre as crianças examinadas, $70,46 \%$ possuíam algum erro refracional, sendo a hipermetropia e o astigmatismo os mais comuns (encontrado em 45,45\% e 10,23\% dos alunos examinados, respectivamente). A miopia foi o erro refracional menos prevalente, presente em 10,23\% dos escolares atendidos.Conclusão: $\mathrm{O}$ estudo mostrou prevalência de 3,92\% de erros refrativos na população de escolares estudada, embora a abstenção elevada possa estar subestimando esse percentual. Percebe-se a necessidade da realização de programas que enfoquem uma maior adesão dos alunos ao encaminhamento, na tentativa de evitar o não diagnóstico de problemas oculares facilmente tratáveis na infância.

Descritores: Erros de refração/epidemiologia; Acuidade visual; Saúde escolar; Estudantes; Prevalência

\footnotetext{
'Doutor, Professor de Oftalmologia da Universidade de Passo Fundo - (RS), Brasil;

${ }^{2}$ Acadêmicos da Faculdade de Medicina da Universidade de Passo Fundo - (RS), Brasil.

Trabalho realizado na Instituição: Faculdade de Medicina da Universidade de Passo Fundo - (RS), Brasil.
} 


\section{INTRODUÇÃO}

A visão é responsável pela maioria de nossas impressões sobre o mundo e nossas memórias dele $^{(1)}$, sendo também essencial para o aprendizado intelectual, e pela maior parte da informação sensorial que recebemos do meio externo. A integridade desse meio de percepção é indispensável para o ensino da criança. Com o ingresso na escola, passamos a desenvolver mais intensamente as atividades intelectuais e sociais, diretamente associadas às capacidades psicomotoras e visuais ${ }^{(2)}$.

A Organização Mundial da Saúde estima que 45 milhões de pessoas são cegas e ao menos 135 milhões têm incapacidade visual, considerando acuidade visual menor que $0,3(6 / 18)$. Entretanto, $80 \%$ dessas baixas visuais são preveníveis ou tratáveis. Projeções estimam que esses números se elevarão a 75 milhões de cegos e 200 milhões de deficientes visuais no ano de 2020 a menos que sejam tomadas ações coordenadas urgentes ${ }^{(3-5)}$.

Essa situação se agrava ainda mais nos países em desenvolvimento, que concentram $90 \%$ das pessoas afetadas pela cegueira e visão subnormal, trazendo profundas conseqüências humanas e socioeconômicas ${ }^{(4,6)}$.

Estudos recentes confirmam a existência de grande número de casos de refrações incorretas, sendo constatado que intervenções corretivas são custo-efetivas e afetam positivamente o desenvolvimento econômico e a qualidade de vida ${ }^{(4)}$.

A quase totalidade das crianças em idade escolar nunca passou por exame oftalmológico. Estima-se que $10 \%$ dessas crianças necessitam de óculos e 10\% apresentam outro problema oftalmológico ${ }^{(7)}$.

Segundo dados do Ministério da Educação, anualmente matriculam-se no país cerca de 5.800 .000 crianças na $1^{a}$ série do ensino fundamental, em escolas públicas, sendo que em cada 1.000 escolares do ensino fundamental, 100 são portadores de erros de refração necessitando de correção (hipermetropia, principalmente; astigmatismo e miopia) $)^{(7.9)}$.

A investigação da prevalência das causas de disfunção visual permite um melhor planejamento de programas oftalmológicos preventivos. A identificação precoce dos problemas oculares na criança, como os erros de refração e o estrabismo, contribui para a prevenção dos danos permanentes à visão binocular ${ }^{(6)}$. Além disso, o retardo no atendimento às crianças é particularmente danoso pelo atraso ou mesmo déficit irreversível que a criança deficiente visual pode sofrer não sendo estimulada, educada e/ou reabilitada precocemente ${ }^{(10)}$.
É, ainda, imprescindível a implementação de programas públicos de saúde ocular para reduzir as conseqüências negativas da prevalência de baixa acuidade visual, uma vez que há falhas no diagnóstico precoce, evidenciada pela alta prevalência observada em escolares não usuários de óculos, sendo também constatado falhas na continuidade da assistência oftalmológica ${ }^{(11)}$. A identificação de problemas visuais em escolares, dessa forma, é uma abordagem que visa a impedir a evolução da doença e melhorar o aprendizado e o aproveitamento escolar ${ }^{(2)}$.

Localizada no Nordeste do Estado do Rio Grande do Sul, Passo Fundo é considerada cidade pólo de mais de 100 municípios localizados na região. O CEAE (Centro Especializado de Assistência ao Educando) realiza a triagem oftalmológica da rede estadual de ensino de Passo Fundo desde 1995, encaminhando os casos necessários ao serviço oftalmológico especializado.

O presente estudo tem o objetivo de verificar a prevalência de erros refrativos em estudantes da primeira série do ensino fundamental das escolas públicas estaduais de Passo Fundo - RS; detectar a ametropia mais comum; verificar se o estudante estava sendo submetido a exame oftalmológico pela primeira vez e analisar a prevalência de alunos que comparecem à consulta especializada após serem encaminhados pela triagem. As informações obtidas são indispensáveis ao planejamento de ações preventivas direcionadas à saúde ocular na região.

\section{Métodos}

A pesquisa consta de um estudo de delineamento transversal para detectar a prevalência de erros refrativos em escolares da cidade de Passo Fundo - RS. As crianças, estudantes da primeira série do ensino fundamental da rede pública estadual, foram examinadas no ano de 2004.

O protocolo da pesquisa foi aprovado pelo Comitê de Ética em Pesquisa da Universidade de Passo Fundo(UPF).

Seguindo o artigo $1^{\circ}$ da lei $\mathrm{n}^{\circ} 10.538$, de 12 de setembro de 1995 , que torna obrigatória a realização do exame oftalmológico nas escolas públicas do estado, o CEAE realiza a triagem na região Nordeste do Rio Grande do Sul. Esta é realizada por médicos clínicos gerais, enfermeiros ou professores devidamente treinados e capacitados, atendendo preferencialmente aos alunos da pré-escola e primeira série do ensino fundamental.

No ano de 2004, foram avaliadas 1608 crianças da primeira série em 26 escolas. 
Como esta é uma atividade de promoção e prevenção à saúde realizada rotineiramente em todas as escolas estaduais de ensino fundamental do município, e o estudo foi realizado com a análise dos prontuários, não foi entregue termo de consentimento aos pais.

O exame oftalmológico padrão foi realizado nas escolas, conforme determinação da Norma Técnica e Operacional para Controle dos Distúrbios da Acuidade Visual em Alunos da Escola Pública ${ }^{(12)}$ e incluiu: exame externo, motilidade ocular, convergência e acuidade visual sem e com correção.

Para a realização da medida da acuidade visual foi utilizada a tabela optométrica de Snellen. A uma distância de cinco metros da tabela as crianças foram colocadas de modo que seus olhos ficassem ao nível das linhas correspondentes a acuidade visual $2 / 3$ ou 1 , sendo cada olho analisado separadamente. As crianças que usavam correção foram examinadas com e sem óculos.

De acordo com o Programa de Saúde Escolar ${ }^{(12)}$, os critérios para encaminhamento foram:

acuidade visual inferior a $2 / 3$, em um ou ambos os olhos, com ou sem sintomas e/ou sinais, em crianças de qualquer idade;

acuidade visual igual a $2 / 3$, em um ou ambos os olhos, com ou sem sintomas e/ou sinais, em crianças com mais de 10 anos de idade;

acuidade visual normal, em ambos os olhos, mas com sintomas e/ou sinais sugestivos de dificuldade visual, em crianças de qualquer idade;

estrabismo.

Dessas crianças triadas, as com dificuldades visuais foram encaminhadas para consulta oftalmológica padrão, realizada por médicos oftalmologistas. O exame oftalmológico foi realizado sem custos para os pais ou responsáveis.

Foram compilados os dados (fornecidos pelo CEAE e pelo atendimento especializado) dos escolares da primeira série das escolas públicas estaduais de Pas-

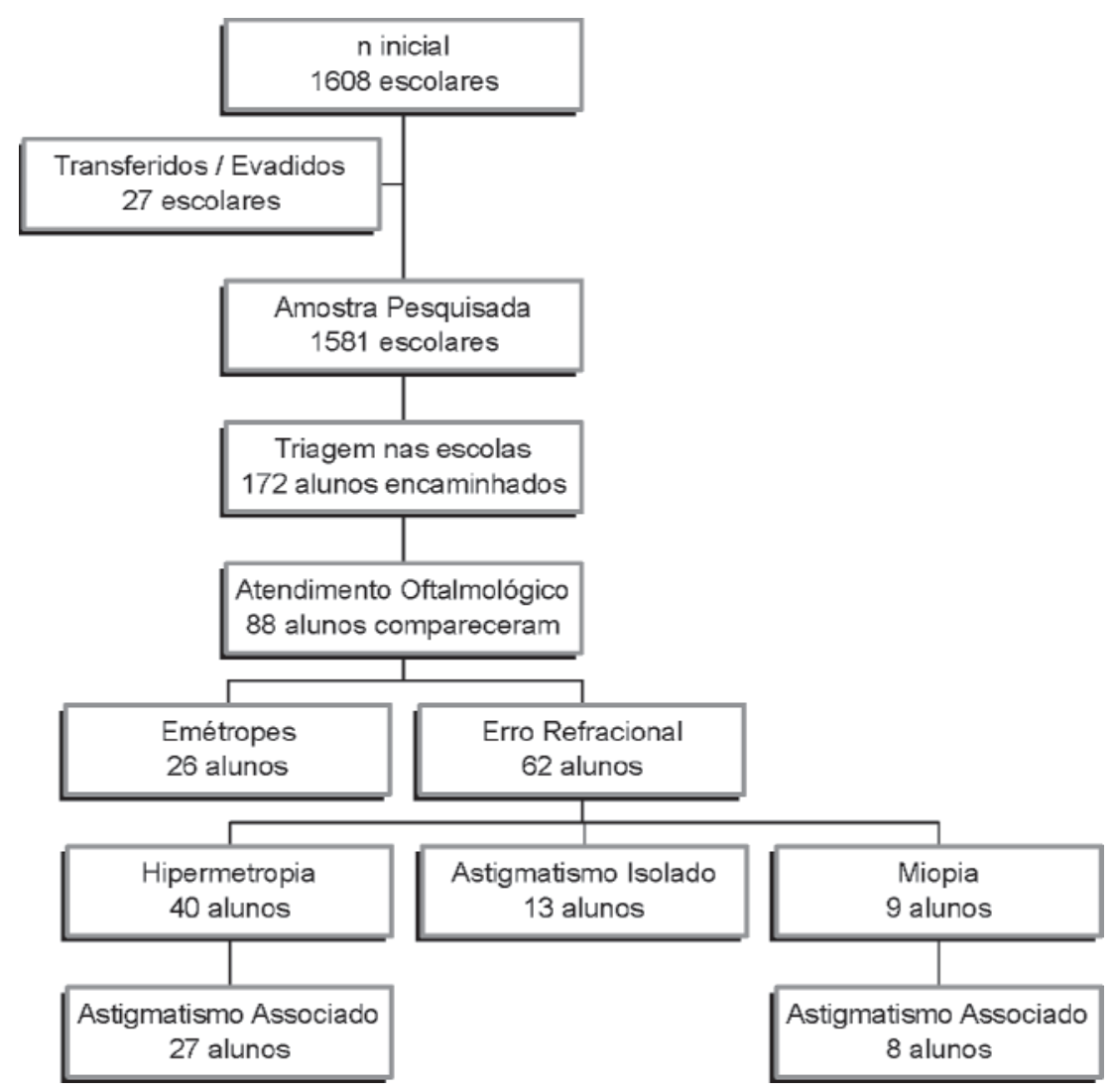

Figura 1: Seguimento dos participantes durante triagem nas escolas, encaminhamento ao especialista e comparecimento à consulta oftalmológica; ametropias encontradas nos alunos atendidos 
Tabela 1

Transtornos de refração observados nos escolares da $1^{\text {a }}$ série das escolas estaduais de Passo Fundo atendidos em consulta oftalmológica no ano de 2004

\begin{tabular}{lrr}
\hline CID 10 (H52)* & n & \% \\
\hline Hipermetropia & 40 & 45,45 \\
Miopia & 9 & 10,23 \\
Astigmatismo & 13 & 14,77 \\
Ambliopia (ansiometropia) & 0 & 0 \\
Sem alterações (emétropes) & 26 & 29,54 \\
Total & $\mathbf{8 8}$ & $\mathbf{1 0 0}$ \\
\hline
\end{tabular}

*Nomenclatura de acordo com o CID-10 - Classificação Estatística Internacional de Doenças e Problemas Relacionados à Saúde - Décima Revisão.

so Fundo no ano de 2004. Analisou-se idade, sexo, se era a primeira consulta ao oftalmologista, acuidade visual, refração e a conduta médica. Os dados coletados foram processados e analisados utilizando-se o programa Microsoft Office - Excel for Windows .

\section{Resultados}

Foram analisadas 26 escolas da rede estadual, sendo que uma escola e uma turma de outra escola foram excluídas do estudo por não terem fornecido o registro da avaliação visual dos escolares. Do total de 1608 crianças submetidas ao exame de triagem, 27 foram perdidas para o estudo por terem sido transferidos da escola ou por evasão, resultando em uma amostra válida de 1581 alunos, sendo 53,01\% (854) pertencentes ao sexo masculino.

Da amostra total, $172(10,88 \%)$ apresentaram baixa de acuidade visual ou algum sintoma de proble- ma visual, sendo encaminhados para atendimento oftalmológico. No entanto, apenas $88(51,16 \%)$ dos encaminhados compareceram à consulta na data e hora marcadas, o que corresponde a 5,56\% do total de alunos pesquisados.

Na ocasião do exame, 72 alunos ( $81,81 \%$ daqueles atendidos pelo oftalmologista) relataram ser aquela sua primeira consulta oftalmológica. Os escolares encontravam-se numa faixa etária entre 6 e 14 anos, sendo a média de idade de 7,10 anos $(\mathrm{DP}=1,38)$. Das crianças examinadas, 46 (52,27\%) eram do sexo feminino.

Entre as crianças examinadas, $70,46 \%$ possuíam algum erro refracional. A hipermetropia foi o mais comum, sendo encontrado em 40 alunos (45,45\%), sendo que 27 destes alunos tinham astigmatismo associado. Astigmatismo isolado foi o segundo erro refracional mais comum, encontrado em 13 alunos (14,77\%). A miopia foi o erro refracional menos prevalente, presente em 9 escolares $(10,23 \%)$, sendo que 8 destes míopes apresentavam astigmatismo associado (Figura 1; Tabela 1).

Em 50\% dos atendimentos (44 alunos), não foram prescritos óculos, seja pelo aluno não possuir erros de refração $(59,09 \%)$ ou por apresentarem alteração de grau igual ou menor que meia dioptria (40,1\%). Já em $36,36 \%$ dos casos atendidos foram prescritas lentes corretivas, e em $11,36 \%$ foi orientada a manutenção da correção em uso. Em 2,27\% dos casos ( 2 alunos), foi orientada a correção cirúrgica de estrabismo (Figura 2).

\section{DısCUSSÃo}

A amostra final de 1581 alunos se denota representativa da população escolar de primeira série das escolas estaduais de Passo Fundo, uma vez que representa 98,32\% dos escolares dessas turmas no ano de 2004.

Foi observado que $10,88 \%$ dos alunos atendidos

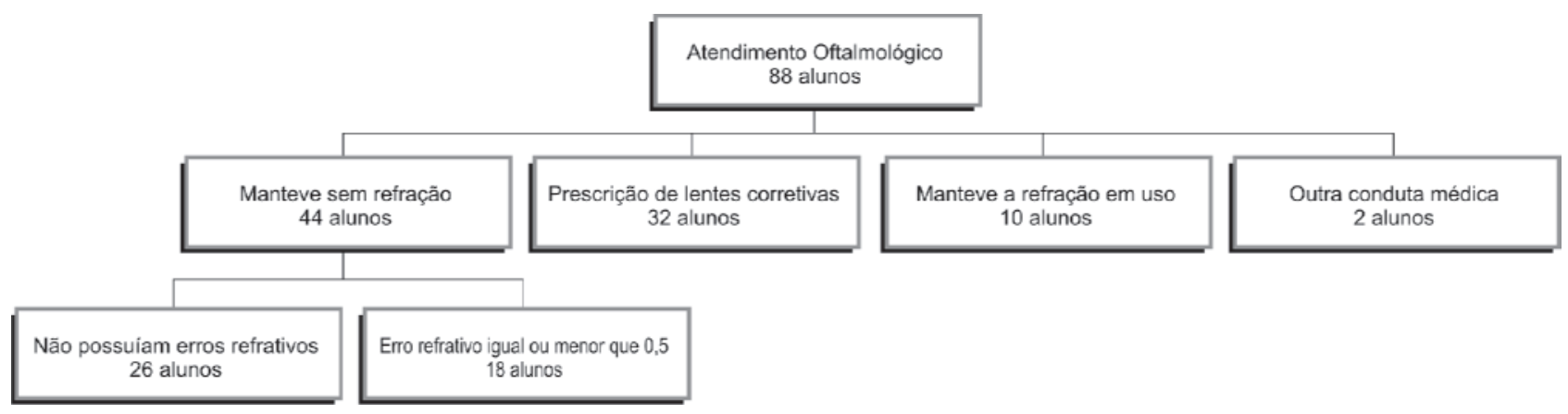

Figura 2: Condutas médicas tomadas após atendimento especializado 
durante a triagem receberam o encaminhamento ao médico oftalmologista. Outros trabalhos realizados em turmas de primeira série do ensino fundamental relatam valores de $15,1 \%{ }^{(2)}$ e $17,1 \%{ }^{(9)}$ de encaminhamentos, mostrando que o número encontrado em Passo Fundo foi relativamente mais baixo que o encontrado em outros locais do país. Entretanto, a metodologia usada para a realização da triagem e encaminhamento ao serviço especializado difere entre os estudos, o que prejudica a comparação entre eles. Além do mais, os profissionais podem ter diferentes formas de realização do exame, o que leva a diferenças estatísticas quando em debate os resultados de alterações visuais, que apresentam grandes variações de prevalência ${ }^{(11)}$. Em estudo realizado em Pernambuco, no ano de $2001^{(6)}$, os erros refrativos foram diagnosticados em $63,9 \%$ da população alvo. Essa alta prevalência se deve ao fato de os componentes da amostra terem procurado atendimento especializado por apresentarem algum sintoma de alteração visual, diferindo do presente estudo, que avaliou alunos que não apresentavam necessariamente queixas visuais.

Dos alunos encaminhados $(10,88 \%)$, somente $51,16 \%$ compareceram à consulta oftalmológica, mesmo sendo um atendimento de referência e gratuito, apresentou uma abstenção de mais de $48 \%$. Esse alto índice de abstenção ao atendimento também foi verificado em outros locais do país, como em Londrina (24,6\% na rede pública e $30,6 \%$ na rede privada $)^{(9)}$, Sorocaba $(11,9 \%)^{(11)}$ e São Paulo (mais de $50 \%)^{(13)}$. Isso pode ser atribuído a diversos fatores, como a falta de consciência da importância de submeter as crianças a um atendimento oftalmológico por parte dos pais ou responsáveis ${ }^{(9)}$, a dificuldade de transporte, pouca orientação e perda do dia de trabalho, como relatado por pais dos escolares no município de São Paulo ${ }^{(13)}$. Isso pode acarretar em perdas tanto para as crianças quanto para o sistema público de saúde, uma vez que é muito mais fácil e barato corrigir os problemas oculares antes que eles avancem para algo mais grave e irrecuperável, haja vista que mais de $90 \%$ dos problemas oftalmológicos podem ser evitados ou minorados com ações preventivas simples, além de constituir uma importante contribuição no combate aos elevados índices de repetência e evasão que tanto castigam a $1^{\text {a }}$ série $^{(7.8)}$. Além disso, essa elevada taxa de absenteísmo pode ser responsável por uma subestimação do resultado, caso se tenha ausentado no dia da avaliação uma proporção maior de indivíduos com baixa acuidade visual ${ }^{(11)}$.

Dos alunos atendidos em serviço especializado, $81,81 \%$ relataram ser aquela sua primeira consulta oftalmológica, valor semelhante ao encontrado em al- guns estudos, que relatam $85,5 \%{ }^{(9)}$ e $67,8 \%{ }^{(13)}$ de primeiras consultas de alunos de escolas públicas. Esse alto índice encontrado denota a necessidade da conscientização dos pais ou responsáveis quanto à procura de atendimento especializado precocemente, antes mesmo da idade escolar e independente da participação da escola, na tentativa de evitar complicações de doenças oculares facilmente tratáveis ${ }^{(9)}$.

Entre os escolares atendidos, $70,46 \%$ tinham reais alterações refracionais, sendo que os $29,54 \%$ restantes eram emétropes. Esse valor de alterações visuais representa $3,92 \%$ da população total estudada, um valor relativamente baixo, ao se considerar que o Ministério da Saúde estima valores em torno de $10 \%$ de escolares do país que necessitam correção refracional com lentes ${ }^{(7.8)}$. Em estudo realizado em Londrina $-\mathrm{PR}^{(9)}$ foi constatado que $37,6 \%$ dos alunos das escolas estaduais, encaminhados ao serviço oftalmológico, apresentavam erro refracional, o que representa 4,86\% do total estudado. Já em Ibiporã, também no Paraná, foram encontrados $31,23 \%$ dos escolares encaminhados com alterações refracionais, representando $4,56 \%$ da amostra ${ }^{(14)}$. Considerando um contexto mundial, a prevalência dos erros refrativos denota-se ainda menor na população de escolares, dependendo do local estudado, sendo apresentadas taxas de 1,01\% na Tanzânia ${ }^{(15)}$ e 1,6\% na Coréia do Sul ${ }^{(16)}$.

$\mathrm{O}$ erro refracional mais comum entre os escolares foi a hipermetropia, encontrada em $45,45 \%$ dos alunos, seguido de astigmatismo isolado $(14,77 \%)$ e miopia $(10,23 \%)$. O astigmatismo também foi encontrado associado à hipermetropia e miopia, respectivamente em $30,68 \%$ e $9,09 \%$ do total de estudados. Essa seqüência de prevalência de erros refracionais se repete em diversos trabalhos ${ }^{(7-8,13,17)}$, sendo que em alguns a miopia se mostra mais prevalente que o astigmatismo isolado ${ }^{(6,14)}$. Garcia et al..$^{(17)}$ mostraram que $79,15 \%$ apresentavam hipermetropia com ou sem associação com astigmatismo, valor alto quando comparado ao presente estudo, que encontrou $45,45 \%$ de prevalência. Contudo, Schimiti et al..$^{(14)}$ encontraram um valor mais próximo ao encontrado em Passo Fundo, de 46,2\%.

Em estudo realizado em Seul, na Coréia do Sul ${ }^{(16)}$, os distúrbios refracionais mais comuns foram o astigmatismo miópico e o astigmatismo hipermetrópico, enquanto na Tanzânia se observou que quase todos os alunos com erro refracional significativo eram míopes $^{(15,18)}$, o que, em análise comparativa com o Brasil, mostra que existem muitas alterações quanto à prevalência desses distúrbios nas diferentes populações mundiais.

Em relação à conduta médica após o exame, 50\% 
dos escolares foram orientados a manter-se sem uso de refração. Destes, 59,09\% eram de fato emétropes, enquanto os $40,9 \%$ restantes possuíam um erro refrativo igual ou menor que 0,5 dioptrias, não sendo necessária a correção. Isso se deve ao fato de que a hipermetropia débil é considerada fisiológica, motivo pelo qual as hipermetopias de $+1,00$ dioptrias ou menores não devem ser corrigidas na criança, assim como no caso de um astigmatismo bem tolerado, sem manifestações funcionais, onde se torna dispensável a correção com lentes ${ }^{(19)}$.

Programas de prevenção aos distúrbios da visão mostram-se efetivos na medida em que detectam precocemente alterações oculares nos escolares, permitindo adequadas formas de correção e tratamento de tais transtornos e melhorando o aprendizado dessas crianças. Esses programas devem ser valorizados pelo fácil acesso à população, fato comprovado pelo baixo custo conferido aos órgãos de saúde pública no atendimento precoce, $\mathrm{o}$ que certamente dispensa gastos excessivos posteriores com problemas oftalmológicos. Não se pode deixar de salientar que o comprometimento visual acarreta restrições de ordem social, econômica e ocupacional.

Os pais representam uma importante contribuição ao processo de detecção de distúrbios oftalmológicos, por isso precisam ser orientados a respeito da importância do processo de saúde ocular, incentivando a criança ao comparecimento no atendimento. Isso só será possível se houver uma conscientização, realizada através de campanhas que envolvam os pais, ou esclarecimentos prévios ao encaminhamento dos escolares, para que não ocorra um grau de abstenção tão grande quanto foi detectado em nosso estudo.

\section{Conclusão}

O estudo mostrou prevalência de 3,92\% de erros refrativos na população de escolares de primeira série das escolas estaduais em Passo Fundo no ano de 2004, embora a abstenção elevada possa estar subestimando esse percentual. Percebe-se a necessidade da realização de programas que enfoquem uma maior adesão dos alunos ao encaminhamento, na tentativa de evitar o não diagnóstico de problemas oculares facilmente tratáveis na infância.

\section{Abstract}

Objectives: To describe and analyze the prevalence of refractive errors among first-graders in state schools of Passo Fundo, main city of the northeast region of Rio Grande do Sul State. Methods: Cross-sectional study of 1608 first- graders in 26 elementary state schools of Passo Fundo, evaluated in 2004. The data were collected by the CEAE (Specialized Center for Student Assistance), which was in charge of the screening at the schools, and by the specialized service, to which those students with abnormal test results were referred.Results: 172 (10.88\%) of the sampled individuals showed poor visual acuity or some symptoms of visual disorders, and were then referred for specialized ophthalmic examination. However, only $88(51.16 \%)$ of the referred patients turned up for the examination. The mean age was 7.10 years ( $D P=1,38)$. Among all evaluated, $70,46 \%$ of them had some refractive disorder, of wich hyperopia and astigmatism were the most frequent ones, having been reported in $45.45 \%$ and $10.23 \%$ of the children, respectively. The myopia were the less frequent refractive disorder, reported in 10,23\% of the school children who turned up for examination. Conclusion: The study showed a prevalence of $3,92 \%$ of refractive errors in the population studied, although the high abstention can be underestimating this percentage. We can realize, from that on, the need of the creation of programs focused in a bigger adhesion of the students to the reference to a specialized service, in the attempt of avoiding the lack of diagnosis of easily treatable disorders in childhood.

Keywords: Refractive errors/epidemiology; Visual acuity; School health; Students; Prevalence

\section{Agradecimentos}

A assistente social Marilene Gatto Bizzotto, do Centro Especializado de Assistência ao Educando Protásio Alves, pelo fornecimento de dados para a execução do presente trabalho.

\section{RefERÊNCIAS}

1. Kandel ER, Schwartz JH, Jessel TM, editores. Princípios da neurociência. 4a ed. Barueri. SP: Manole; 2003.

2. Granzoto JA, Ostermann CSPS, Brum LF, Pereira PG, Granzoto T. Avaliação da acuidade visual em escolares da $1^{\text {a }}$ série do ensino fundamental. Arq Bras Oftalmol. 2003; 66(2):167-71.

3. World Health Organization. Strategies for the prevention of blindness in national programmes: a primary health care approach. 2nd ed. Geneva: World Health Organization; 1997.

4. World Health Organization. Fifty-sixth World Health Assembly (A56/26): Elimination of avoidable blindness. Geneva; WHO; 2003.

5. Gilbert C, Foster A. Childhood blindness in the context of VISION 2020 - the right to sight. Bull World Health Organ. 2001; 79(3):227-32.

6. Albuquerque RC, Alves JGB. Afecções oculares prevalentes em crianças de baixa renda atendidas em um serviço oftalmológico na cidade do Recife - PE, Brasil. Arq Bras Oftalmol. 2003; 66(6):831-4. 
7. Alves MR, Kara-José N. Manual de orientação. Campanha Veja Bem Brasil. São Paulo: Conselho Brasileiro de Oftalmologia; 1998.

8. Alves MR, Kara-José N. Campanha Nacional de Reabilitação Visual: Manual de Orientação. Conselho Brasileiro de Oftalmologia. São Paulo: CBO; 1999.

9. Lopes GJA, Casella AMB, Chuí CA. Prevalência de acuidade visual reduzida nos alunos da primeira série do ensino fundamental das redes pública estadual e privada de Londrina-PR, no ano de 2000. Arq Bras Oftalmol. 2002; 65(6):659-64.

10. Kara José N, Carvalho KMM, Pereira VL, Venturini NHB, Gasparetto MEFR, Gushiken MT. Estudo retrospectivo dos primeiros 140 casos atendidos na clínica de visão subnormal do Hospital de Clínicas da Unicamp. Arq Bras Oftalmol. 1988; 51(2):65-9.

11. Gianini RJ, Masi E, Coelho EC, Oréfice FR, Moraes RA. Prevalência de baixa acuidade visual em escolares da rede pública, Sorocaba. Rev Saúde Pública = J Public Health. 2004; 38(2):201-8.

12. Moreira AMM, Garcia CP, Scherek CN, Martins CC, Telichevski N, Picon PD, et al. Programa de Saúde Escolar: Controle dos distúrbios da acuidade visual na escola pública [Normas Técnicas e Operacionais]. 2a ed. Rio Grande do Sul: Secretaria Estadual da Saúde; 1999.

13. Alves MR, Temporini ER, Kara-José N. Atendimento oftalmológico de escolares do sistema público de ensino no município de São Paulo: aspectos médicos-sociais. Arq Bras Oftalmol. 2000; 63(5):359-63.

14. Schimiti RB, Costa VP, Gregui MJF, Kara-José N, Temporini ER. Prevalence of refractive errors and ocular disorders in preschool and schoolchildren of Ibiporã - PR, Brazil (1989 to 1996). Arq Bras Oftalmol. 2001; 64(5):379-84.
15. Wedner SH, Ross DA, Balira R, Kaji L, Foster A. Prevalence of eye diseases in primary school children in a rural area of Tanzania. Br J Ophthalmol. 2000; 84(11):1291-7.

16. Lim HT, Yu YS, Park SH, Ahn H, Kim S, Lee M, et al. The Seoul Metropolitan Preschool Vision Screening Programme: results from South Korea. Br J Ophthalmol. 2004; 88(7):929-33. Comment in: Br J Ophthalmol. 2005; 89(3):392-3.

17. Garcia CAA, Oréfice F, Nobre GFD, Souza DB, Rocha MLR, Vianna RNG. Prevalence of refractive errors in students in Northeastern Brazil. Arq Bras Oftalmol. 2005; 68(3):321-5.

18. Wedner SH, Ross DA, Todd J, Anemona A, Balira R, Foster A. Myopia in secondary school students in Mwanza City, Tanzania: the need for a national screening programme. Br J Ophthalmol. 2002; 86(11):1200-6. Comment in: Br J Ophthalmol. 2003; 87(5):660.

19. Gil del Rio E. Óptica fisiológica clínica: refracción. 4a ed. Barcelona: Toray; 1981.

\section{ENDEREÇO PARA CORRESPONDÊNCIA: Leandro Mazzoleni Stramari Rua Teixeira Teixeira de Freitas, $\mathbf{n}^{\circ} 231$ CEP 99025-140 - Passo Fundo - RS \\ Fone: (54) 9184-2871 \\ E-mail: leandrostramari@yahoo.com.br}

